\title{
RENDRE SON HISTOIRE À LA MYSTIQUE RENDRE LA MYSTIQUE À L'HISTOIRE
}

Jean BARUzI, L'Intelligence mystique, Paris, Berg international, 1986.

Michel DE CeRTEAU, "Historicités mystiques", Recherches de science religieuse, Paris, t. 73, 1985, p. 325-353.

Michel DE CERTEAU, La Fable mystique, Paris, Gallimard, “ Bibliothèque des histoires ", 1982 ; $2^{\mathrm{e}}$ éd., La Fable mystique. T. 1 : XVT-XVIr siècle, Paris, Gallimard, « Tel », 1987.

Luce Giard, sous la dir. de, Le Voyage mystique. Michel de Certeau, Paris, Recherches de science religieuse/Cerf, 1988.

« La mystique, en tant que vie, aboutit à des individus, et à eux seuls ", écrivait Jean Baruzi dans son Saint Jean de la Croix et le problème de l'expérience mystique $^{1}$. Il pose ainsi, commente J.-L. Vieilliard-Baron qui a édité et préfacé le recueil de textes, L'Intelligence mystique, «le principe métaphysique (je souligne) qui commande sa méthode ${ }^{2}$. Dans l'article «Historicités mystiques", M. de Certeau, qui s'intéresse surtout aux Deux sources de Bergson, range l'œuvre de J. Baruzi parmi les autres où « se retrouve à l'évidence, le même fonctionnement "philosophique" du mysticisme $"{ }^{3}$.

Une partie de cet article dévoile les conditions de possibilité de l'avènement, au début de notre siècle, d'une intelligence de la mystique, ou plutôt du mysticisme, précisément selon un principe métaphysique. Il les voit dans le développement des sciences sociales et dans la crise de la philosophie qui s'ensuit :

" des phénomènes religieux, distribués en "objets" relevant de disciplines particulières (la critique historique, la pathologie, l'ethnologie, etc.), répartis en aires culturelles et en périodes historiques hétérogènes, se distingue en effet une sorte de " reste" ou de "fond " commun qui reçoit le nom de "mysticisme" (employé déjà depuis plus d'un siècle) ${ }^{4}{ }^{4}$.

1. Jean BARUzI, Saint Jean de la Croix et le problème de l'expérience mystique, $1^{\text {se }}$ éd., Paris, Alcan, 1924, p. II.

2. L'Intelligence mystique, p. 32.

3. « Historicités mystiques ", p. 339.

4. Ibid., p. 337.

Revue de synthèse : IV S. $\mathrm{N}^{\circ} 4$, oct.-déc. 1990. 
Dans la parcellisation des savoirs, ce que les mystiques nommaient leur «science expérimentale " est appelé à remplir une fonction unifiante. Il se légitime au regard des exigences de scientificité justement comme " donnée expérimentale ", et en même temps « permet de surmonter les clôtures créées par l'analyse scientifique entre ses disciplines ou entre ses objets ».

L'intention de l'article de M. de Certeau n'est pas la critique de l'abord de la mystique par des philosophes. Avec l'œuvre de Bergson qui « peut servir d'exemple " et en rassemblant autour d'elle plusieurs noms, dont celui de Baruzi, il restitue en somme l'approche philosophique, caractéristique d'une époque, à son historicité. De même il y restitue l'analyse concomitante que font les nouvelles sciences sociales et humaines du " mysticisme ". Mais le but dernier est de donner à entendre comment la mystique s'inscrit elle-même dans l'histoire, comment ce qu'elle a été dans son temps propre, donne de la lumière sur le recours à elle par d'autres en un autre temps.

Le "vague" parait être le premier obstacle que rencontre toute étude sur la mystique. Souvent chaque auteur se juge obligé de donner préalablement sa définition de la mystique en critiquant la plupart des autres qu'il juge trop larges ou floues. Il ne semble trouver d'abord avec le mot " mystique " qu'une notion et un objet d'étude fuyants. L'éditeur du recueil d'articles de J. Baruzi a mis en tête celui qui s'intitule " De l'emploi légitime et de l'emploi abusif du mot " mystique " ". C'est un des plus récents, il date de 1948, mais il est parfaitement à sa place en premier. Contre Lévy-Bruhl, il écarte l'emploi de l'adjectif «mystique " pour qualifier les sociétés primitives. Et il affirme, ce faisant, que la mystique authentique « est l'une des grandes formes de la pensée ", " aussi rare que la pensée métaphysique "; "la pensée mystique originale " lui est au moins comparable, elle apporte "une nouvelle façon de penser l'absolu». Définie comme haute pensée, la mystique authentique est ainsi distinguée « d'états de vague mysticité ", contre lesquels les mystiques eux-mêmes mettent en garde comme ce qui n'est pas mystique.

L'article suivant, plus ancien, une communication à la Société française de philosophie du 2 mai 1925 pose « le problème de la valeur noétique de l'expérience mystique " et s'interroge sur son pouvoir de préparer à une philosophie de l'Esprit, par les purifications passives et par son orientation « vers le non-voir et le non-savoir". Bref, comme le déclare Baruzi lui-même, "nous ne pouvons éluder le problème de la métaphysique de la mystique ". Les deux tiers du livre, par le sujet de chacun des articles, sont consacrés à ce problème. Et l'un d'eux traite du " point de rencontre de Bergson et de la mystique ». A quelques semaines près, ce recueil de textes de Baruzi a paru en même temps que l'article de $M$. de Certeau, dont il aurait pu nourrir les pages sur la " fonction philosophique » dévolue à la mystique.

C'est en historien que $M$. de Certeau a formulé sa propre interrogation, plus exactement c'est de la mystique qu'il a fait ce qu'il appelle dans les premières lignes d'Écriture de l'histoire , le « site " de son travail. Dans le livre d'hommage,

5. Michel de Certeau, Écriture de l'histoire, Paris, Gallimard, « Bibliothèque des histoires ", 1975, p. 27. 
Le Voyage mystique. Michel de Certeau, deux articles, "Le secret d'un travail ${ }^{6}$ de Jacques Le Brin et "Une histoire en actes " ${ }^{7}$ de Dominique Julia, exposent et analysent sa recherche et son historiographie. Travail et actes, les mots sont caractéristiques, la mystique est moins à entendre comme l'objet d'études historiques successives que comme «lieu » d'une opération incessante de l'historien. Comme les mystiques sont des itinérants, l'historien selon $\mathbf{M}$. de Certeau est à sa façon un infatigable marcheur. La bibliographie, établie par Luce Giard à la fin du volume, précise et complète au moins d'intention, compte 422 numéros, auxquels s'ajoute la liste des comptes rendus bibliographiques. Elle permet de saisir justement comment M. de Certeau travaillait :

« souvent un premier article était repris, remanié, développé, parfois à dix ou quinze ans d'intervalle, pour donner naissance à une nouvelle version, enrichie et purifiée, ou encore il était mis en pièces et des fragments réinvestis ici ou là ${ }^{8}$.

Cette " habitude constante de réemploi » caractérise l'œuvre comme incessant progrès, que la bibliographie détaillée donne à suivre presque pas à pas.

Michel de Certeau a commencé par des travaux d'édition critique, notamment le Mémorial de Pierre Favre, puis des textes de Jean-Joseph Surin, Guide spirituel (1963) (il vient d'être réimprimé en 1989) et le gros volume de Correspondance $(1966)^{9}$.

Ces travaux, « les détours labyrinthiques (et finalement si rusés) de l'édition critique $"{ }^{10}$, lui fournissaient, non seulement une matière, mais encore ouvraient « l'espace " où exercer le métier d'historien et, par-delà, où penser cet exercice. Au cours des années, dans l'écriture de textes qui, pour ainsi dire, se génèrent les uns les autres, lire les mystiques, faire de l'histoire, penser l'histoire se dévoilent être solidement noués entre eux, s'étayer mutuellement, se mettre en cause réciproquement.

Le rend possible la restitution de la mystique à l'historique. Entendons par là une rupture avec l'appréhension de la mystique comme un " reste " transcendant toujours de quelque façon l'histoire, un fond qui tout au long des siècles s'exprimerait dans une infinie variété de discours. La mystique est un discours, et daté. Il apparaît et se développe dans l'Occident chrétien entre le $\mathrm{xv}^{E}$ et le xvir siècle, comme en témoigne d'ailleurs la langue, des spirituels comme de leurs détracteurs : " mystique " jusqu'alors adjectif, devient substantif, la mystique et les mystiques.

Dès lors, la nécessité, pour éviter le vague dont nous parlions, de définir préalablement le sens qu'on donne au terme, n'existe plus, ou plus telle quelle. On détermine le référent historique de la mystique. Parler d'elle c'est alors, d'une cer-

6. P. 77-95.

7. P. 103-123.

8. P. 191-243.

9. Bienheureux Pierre FaVre, Mémorial, Paris, Desclèe de Brouwer, "Christus », 1960; Jean-Joseph SurIN, Guide spirituel pour la perfection, Paris, Desclée de Brouwer, "Christus », 1963 ; ID., Correspondance, Paris, Desclée de Brouwer, " Bibliothèque européenne », 1966.

10. La Fable mystique, p. 19. 
taine façon, ne pas cesser de la définir, travailler les textes pour saisir comment ils la produisent. Ce n'est pas chercher l'ineffable que ces textes abriteraient, mais découvrir et montrer comment le discours l'opère. Dans le langage même des Xvi ${ }^{e}$ et $\mathrm{xvII}^{\mathrm{e}}$ siècles, répète $\mathrm{M}$. de Certeau, on appelle mystique le discours, l'expérience est qualifiée de spirituelle.

Ainsi en histoire de la mystique, de la "science des saints ", il n'est pas possible de raisonner en terme d'une épistémologie de l'objet et de la méthode. Il y a une "manière " mystique, non une matière définie ou définissable, une " manière, conclut l'article " Historicités mystiques", d'opérer ou de dire ". La mystique, selon l'expression favorite de $M$. de Certeau, est un "modus loquendi ».

Les textes des mystiques ou de leurs commentateurs - par exemple, Diego de Jésus voulant "donner une plus facile intelligence des phrases mystiques" de Jean de la Croix - l'indiquent très clairement à qui sait les lire. Mystiques sont bien moins des pensées que des tours, des tournures, bref des façons de " tourner » le langage qu'on appelle aussi des « tropes », parmi lesquels comme figure principale l'oxymoron. L'historien, sur ce point, fait une ample moisson de citations, sans pouvoir prétendre être exhaustif, car c'est toute la littérature mystique qui se qualifie comme telle par sa façon de dire. Une sorte de contre-épreuve est fournie par les adversaires des mystiques. Rien qu'en France, depuis les moqueries du P. Binet, contre les disciples de Denys, jusqu'au Dictionnaire de Trévoux, pour qui Desmarets a perdu l'esprit en écrivant de la " mystiquerie ", ce sont les " exagérations », les jeux de mots, les formules absconses, un jargon qui sont dénoncés. On ne peut saisir ce que le discours mystique veut dire.

Mais sans doute ne veut-il rien dire. D'ailleurs, il n'y a pas de discours mystique propre. Il se caractérise bien plutôt comme une façon de s'emparer d'autres discours, théologique, ascétique, autobiographique, poétique pour, en le " tournant " autrement, le faire, si l'on ose dire, " déparler ". La phrase mystique, écrit M. de Certeau, est un "artefact » du silence. Il fait comme tenir au discours apparent un discours secret, à celui qui s'écoute ou se lit ce qui ne s'entend pas, la parole de «l'autre".

L'historien ne peut se limiter à une analyse des discours. Il a pour dessein et fin de saisir les conditions de leur apparition à un moment historique donné. Court, comme un fil directeur tout au long de La Fable mystique et en suture les chapitres, l'idée de « délaissement ».

Dès l'introduction, un paragraphe situe les mystiques dans « la tradition humiliée " et le livre s'achève par un chapitre sur le nomadisme de Labadie. Le délaissement c'est d'abord le fait d'une situation : l'appartenance des mystiques «le plus souvent à des régions et des catégories en voie de récession socio-économique, défavorisées par le changement, marginalisées par le progrès ou ruinées par les guerres ". Ils vivent l'effritement de leurs assises sociales, l'incertitude du présent et l'absence d'avenir, bref, de par leur origine familiale et locale, ils souffrent souvent une perte. Perte endurée aussi dans les familles religieuses quand ils y appartiennent : déception des institutions décadentes, frustration d'un idéal religieux espéré. Jean de la Croix combat pour que l'ordre séculaire des carmes retrouve sa pureté. Mais un ordre récent comme la Compagnie de Jésus. est lui-même en crise au moment où son fondateur est béatifié (1609), puis cano- 
nisé (1612). Une semblable revendication de pureté spirituelle s'y fait alors entendre de la part de jeunes jésuites, notamment en Aquitaine, la province du P. Surin.

Par-delà, la perte du sens est celle de l'institution chrétienne tout entière : de l'organisation ecclésiale, de la symbolique cultuelle, de la tradition doctrinale. Tout est ruines, mais dans ces ruines les mystiques campent, comme si le détruit était leur héritage et que le pire devait être pour eux le plus sûr.

Dès lors, pour y revenir, le recours à la mystique par la philosophie laisse entendre, selon l'historien, autre chose que ce que disent explicitement les philosophes. Leur recherche d'un "principe métaphysique ", tiré de l'expérience des grands spirituels réfère sans qu'ils le sachent, ou du moins sans qu'ils le donnent à entendre clairement, leur propre deuil à un autre deuil, le délaissement philosophique au délaissement plus ancien du théologique. Plus qu'un souhait réalisable "le supplément d'âme " réclamé par Bergson exprime une perte et pointe le «non-lieu " de la philosophie, comme trois siècles plus tôt la quête et l'exigence d'un renouveau par l'intériorité et le pur spirituel désignaient le site déserté du religieux. Et la recherche baruzienne de l'intelligence de la mystique par monographies, parce qu'elle est toujours individuelle et singulière, est aussi une sorte d'aveu implicite d'absence de champ philosophique.

L'historien de la mystique définit donc celle-ci d'abord comme formation historique. Elle a un commencement et une fin. Elle a son époque et son aire. L'usage général du mot qui en fait une sorte de constante à la fois transtemporelle et transreligieuse est de soi et d'emblée exclu. Mais cet usage n'est pas inexplicable, ni plus largement la polysémie du terme que paraît révéler la variété de ses emplois au cours des temps. L'étude circonscrite de la mystique proprement dite des $X^{e}{ }^{e}$ et $X I^{e} I^{e}$ siècles permet de se reporter aux autres inscriptions du mystique, ou du mysticisme, dans l'histoire. En un mot, si dans la période même de la mystique "moderne " le mot fonctionne comme des guillemets, signale qu'en écrivant on écrit autre chose et que la parole dite parle le non-dit de l'autre, c'est cette fonction qui est appelée à opérer hors du temps de la « science expérimentale ». Avant comme après, car la mystique procède à une lecture spécifique de ses antécédents pour s'y ancrer et s'y reconnaître, particulièrement des œuvres de Denys l'Aréopagite : « le corpus dionysien soutient l'émancipation de cette "science ", dont il reçoit en retour l'interprétation qui le place de plus en plus sous la lumière nocturne de sa Théologie mystique $"{ }^{11}$. Les mystiques se donnent une histoire, ou du moins un passé, une antiquité, une tradition textuelle qui, la légende de Denys aidant, remonterait jusqu'au temps des apôtres. Après eux, des philosophes - et d'autres - leur redonnent un présent comme pour conjurer le leur, la faille qui se produit dans leur propre discipline.

Cette vue des philosophes du $\mathrm{xx}^{e}$ siècle n'est pas celle d'un historien de la philosophie, même si celui-ci aboutit éventuellement à des conclusions analogues. Dans ce cas, l'historien de la mystique a agrandi progressivement son cercle, le champ de sa propre recherche à partir de son travail initial, ce que $\mathbf{M}$. de Certeau appelle son " point aveugle» : ses éditions des textes de Surin. Il est de la sorte

11. Ibid., p. 140 . 
conduit à penser et juger ce que lui-même opère dans son écriture de l'histoire, ou plutôt d'une histoire. Il constate une symétrie entre l'historiographie de la mystique et la mystique elle-même. L'une et l'autre s'écrivent à partir d'une absence, car l'histoire parle de ce qui s'est perdu et ne reviendra plus :

"l'historien des mystiques appelé comme eux à dire l'autre, redouble leur expérience en l'étudiant : un exercice d'absence définit à la fois l'opération par laquelle il produit son texte et celle qui construit la leur. [...] II cherche un disparu qui cherchait un disparu, etc. ${ }^{12}$.

Pour cette raison - je ne retiendrai que ce point, il faudrait aussi souligner les divergences entre la mystique et son histoire - l'historien de la mystique peutêtre éprouve plus qu'un autre une nostalgie. "Il est exilé de ce qu'il traite ", dit la première phrase de La Fable mystique, "l'écriture que je dédie aux discours mystiques de (ou sur) la présence (de Dieu) a pour statut de ne pas en être. Elle se produit à partir de ce deuil, mais un deuil inaccepté, devenu la maladie d'être séparé... " L'écriture de cette histoire-là est, en somme, la nostalgie d'une autre nostalgie, la forme contemporaine de deuil d'un autre deuil. Ainsi, sans aucun doute, une certaine idée de l'histoire et la façon de la faire se prennent du sujet traité. Mais la réciproque est vraie. Au cours du travail une sorte d'interaction entre l'objet de la recherche et l'outil historiographique ne cesse de s'accuser. Et lorsqu'il rencontre des philosophes de la mystique, l'historien qui a avoué la sienne leur fait dire leur nostalgie refoulée.

Un texte de $M$. de Certeau, publié en annexe à la Correspondance de J.-J. Surin, décrit chez Jeanne des Anges, la prieure possédée des ursulines de Loudun, le désir d'être ce qu'elle n'est pas : une mystique. Alors elle joue à l'être. Simulation, dissimulation, hypocrisie ne sont pourtant pas en l'occurrence des termes parfaitement adéquats. Car si Jeanne reconnaît parfois qu'elle donne le change, elle avoue aussi sa médiocrité spirituelle et se trompe souvent elle-même sur elle-même. Une autre forme de nostalgie douloureusement endurée est ainsi révélée qui invite plus à la compassion qu'à la condamnation.

Ce texte a été repris dans une réédition récente de l'Autobiographie ${ }^{13}$ de Jeanne des Anges. La première édition de 1886 était due à deux élèves de Charcot, les docteurs Legué et Gilles de la Tourette. La réédition reprend leur introduction et leurs notes, ainsi que la préface de leur " honoré maître " auquel ils ont dédié le livre. L'ajout, en appendice de l'étude de $\mathbf{M}$. de Certeau, donne à la publication du texte l'intérêt supplémentaire d'une comparaison entre les interprétations à un siècle de distance. Les premiers interprètes, psychiatres, classent et font de Jeanne des Anges un cas exemplaire de "passion hystérique ", auprès duquel celui de Thérèse d'Avila, ajoutent-ils, " pâlit singulièrement ". Michel de Certeau ne pose pas un diagnostic, il analyse les textes et par les textes éclaire les relations de Jeanne notamment avec ses directeurs Surin et Saint-Jure.

12. Ibid., p. 21.

13. Sgur Jeanne des Anges, Supérieure du couvent des ursulines de Loudun (xvi1), Autobiographie d'une hystérique possédée, Montbonnot-Saint-Martin, Jérôme Millon, 1985 (depuis la rédaction de ce texte, les Éditions Jérôme Millon ont donné en 1990 une nouvelle édition corrigée de ce texte). 
Cette réédition est l'œuvre d'un éditeur de l'Isère, Jérôme Millon ${ }^{14}$. Il vient également de publier en 1990 l'ouvrage suivant : Jean-Joseph Surin, Triomphe de l'amour divin sur les puissances de l'Enfer et Science expérimentale des choses de l'autre vie, 1653-1660, suivi de Les Aventures de Jean-Joseph Surin par Michel de Certeau. On lui doit l'édition d'autres livres spirituels : Margery Kempe, Louise du Néant...

C'est l'occasion de signaler, pour finir, que des éditeurs, souvent de petite taille, font paraitre actuellement de nombreux textes mystiques. Regain de faveur de la mystique? Pourquoi en notre temps? De quelle crise contemporaine est-ce l'effet ou le signe? Il faudrait certainement pour trouver réponses à ces questions lire alors les textes où $M$. de Certeau, à la fois analyste et acteur de notre culture présente, a rapporté son "expérience " du "christianisme éclaté ", et finalement y chercher à entendre pourquoi il a dit « inaccepté " son propre deuil de la mystique.

Joseph BEAUDE.

14. Au moment de la parution, à Montbonnot-Saint-Martin, maintenant à Grenoble. 